\title{
Multikinase Inhibitor SAR103168
}

National Cancer Institute

\section{Source}

National Cancer Institute. Multikinase Inhibitor SAR103168. NCI Thesaurus. Code C88278.

A multikinase inhibitor with potential antineoplastic activity. Upon intravenous infusion, multikinase inhibitor SAR103168 may, through the inhibition of multiple kinases, inhibit the phosphorylation and activation of signal transducer and activator of transcription 5 (STAT5). ST AT 5, a protein often upregulated in cancer cells, plays a key role in signal transduction pathways and the suppression of apoptosis. 